\title{
Adding a low-concentration sciatic nerve block to total knee arthroplasty in patients susceptible to the adverse effects of non- steroidal anti-inflammatory drugs (NSAIDs): a randomized controlled trial
}

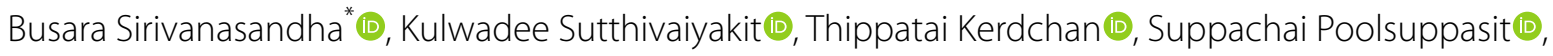
Suwimon Tangwiwat (1) and Pathom Halilamien (1)

\begin{abstract}
Background: This study compared the effects of adductor canal blocks with those of a low concentration of popliteal-sciatic nerve block (SNB) and dexamethasone as an adjunctive technique for total knee arthroplasties (TKA) in patients susceptible to the adverse effects of NSAIDs.

Methods: A prospective, double-blinded, randomized controlled trial was performed in 50 patients susceptible to the adverse effects of NSAIDs undergoing unilateral TKAs. All patients received spinal anesthesia, adductor canal blocks, and periarticular infiltration. The 25 patients in the intervention group received SNB $(0.125 \%$ bupivacaine [20 ml] and dexamethasone [5 mg]).

Results: The SNB group significantly had lower median resting pain scores at 6, 12, and $18 \mathrm{~h}$ : the control group, 1 $(0-4.5), 3(0-5)$, and $3(2-5)$; the intervention group, $0(0-0), 0(0-3)$, and $1(0-3) ; p$-values, $0.012,0.021$, and 0.010 , respectively. Movement-evoked pain scores at 6, 12, and $18 \mathrm{~h}$ were also lower: control group, $3(0-5.5), 5(2.5-6.5)$, and 7 (4-9); intervention group, $0(0-1.5), 2(0-4)$, and $3(2-5)$; $p$-values, $0.019,0.005$, and 0.001 , respectively. There were no differences in motor function. Moreover, the mean morphine consumption $24 \mathrm{~h}$ was also reduced in the SNB group: control group, $3.80 \pm 2.48 \mathrm{mg}$; intervention group, $1.96 \pm 2 \mathrm{mg}$; $p$-value, 0.005 .
\end{abstract}

Conclusion: For patients susceptible to the adverse effects of NSAIDs, a low concentration of SNB and dexamethasone is an effective adjunctive technique for early postoperative pain control (especially on movement) following TKAs, without an increase in motor weakness.

Trial registration: ClinicalTrials.gov, NCT03486548, Registered 3 April 2018.

Keywords: Adverse effects of NSAIDs, Popliteal-sciatic nerve block (SNB), Dexamethasone, Total knee arthroplasty (TKA), Adductor canal blocks, Pain management

*Correspondence: busarasiri@gmail.com

Department of Anesthesiology, Faculty of Medicine Siriraj Hospital, Mahidol University, 2 Wanglang Road, Bangkok Noi, Bangkok 10700, Thailand

\section{Background}

Total knee arthroplasty (TKA), one of the most common surgeries, causes moderate to severe postoperative pain. Multimodal analgesia is currently the standard treatment for acute postoperative pain control, and original author(s) and the source, provide a link to the Creative Commons licence, and indicate if changes were made. The images or other third party material in this article are included in the article's Creative Commons licence, unless indicated otherwise in a credit line to the material. If material is not included in the article's Creative Commons licence and your intended use is not permitted by statutory regulation or exceeds the permitted use, you will need to obtain permission directly from the copyright holder. To view a copy of this licence, visit http://creativecommons.org/licenses/by/4.0/. The Creative Commons Public Domain Dedication waiver (http://creativeco mmons.org/publicdomain/zero/1.0/) applies to the data made available in this article, unless otherwise stated in a credit line to the data. 
it aims to reduce the usage of opioids. The absence of opioid side effects promotes early ambulation, hastens postoperative recovery, decreases the incidence of postoperative complications, and improves patient satisfaction [1].

A peripheral nerve block is a popular technique for acute postoperative pain control after TKA. The sensory nerves that supply the anterior side of the knee originate from branches of the femoral nerve, the lateral femoral cutaneous nerve, and the common peroneal nerve. In comparison, the posterior side of the knee is supplied by the popliteal nerve plexus, which derives from the tibial nerve, and branches of the obturator nerve [2,3]. While postoperative knee pain can be reduced with either an adductor canal block or a femoral nerve block, the former has a lower incidence of quadriceps muscle weakness [4-6].

At our institute, multimodal analgesia is currently utilized for postoperative pain control after TKA. Depending on the judgement of the attending anesthesiologist, the multimodal analgesia comprises a varying combination of spinal anesthesia, adductor canal block, periarticular infiltration, postoperative multimodal medication, and systemic nonsteroidal anti-inflammatory drugs (NSAIDs). The study by Gwam et al. reported an average pain score of 4.94 (range 0-9) for TKA patients whose postoperative pain was being managed with a combination of an adductor canal block and periarticular infiltration [7]. Moreover, periarticular infiltration may only reduce pain for 6 to $12 \mathrm{~h}$ after surgery [8]. Therefore, NSAIDs are the main agents used to relieve pain and reduce opioid consumption.

Patients susceptible to the adverse effects of NSAIDssuch as patients with chronic kidney disease, coronary artery disease, gastric ulcer, a history of stroke, or advanced age-have a limited ability to use systemic NSAIDs postoperatively but can be safe for a local infiltration with NSAIDs has the local rather than systemic effect [9, 10]. Without the opioid-sparing effects of NSAIDs [11-13], patients in this group may suffer from worse postoperative pain levels than previously reported by Gwam et al.

A sciatic nerve block (SNB) is considered to reduce posterior knee pain $[14,15]$. It may cause hamstring muscle weakness or foot drop, resulting in delayed postoperative ambulation [16]. A low concentration of local anesthetic may reduce the risk of a motor block, but it may also affect its analgesic duration. However, a systematic review and meta-analysis reported that adding dexamethasone to the local anesthetic drug injected into nerve areas can increase the analgesic duration of the local anesthetic without causing severe side effects [17, $18]$.
The primary purpose of this study was to evaluate the benefit of overall postoperative pain control by adding a combination of a low concentration of popliteal-SNB and dexamethasone as an adjunctive technique for TKA in patients susceptible to the adverse effects of NSAIDs. In addition, it compared their effects with those of an adductor canal block (ACB) and periarticular infiltration analgesia (PIA) for such patients.

\section{Methods}

\section{Study design and population}

Prior to commencement, this single-center, prospective, double-blinded, randomized controlled trial was approved by the Siriraj Institutional Review Board (COA no. Si123/2018) and registered at ClinicalTrials.gov, NCT03486548, Registered 3 April 2018, https://clini caltrials.gov/ct2/show/NCT03486548. The investigation was conducted on patients aged 50-85years; had American Society of Anesthesiologists physical statuses of I-III; and were scheduled to undergo elective, unilateral, primary TKA at Siriraj Hospital May 2018-December 2019. The inclusion criteria were patients susceptible to the adverse effects of NSAIDs: history of chronic kidney disease (estimated glomerular filtration rate $<50 \mathrm{ml} /$ $\min / 1.73 \mathrm{~m}^{2}$ ), coronary artery disease, cerebrovascular disease, gastric ulcer, or an allergy to NSAIDs other than ketorolac. The exclusion criteria were a bodyweight of $<45 \mathrm{~kg}$; allergy to bupivacaine, ketorolac, or dexamethasone; uncontrolled diabetes; contraindication to regional anesthesia; cognitive impairment; preexisting neuropathy; a neurological deficit in the lower extremities; and preexisting pain requiring the chronic use of oral morphine $(20 \mathrm{mg} /$ day or equivalent). Patients who declined to participate were also excluded.

\section{Randomization and blinding}

The patients $(n=50)$ were randomized into an intervention group and a control group in blocks of four. This was achieved by using the sealed-envelope technique and computer-generated randomization lists sourced from an internet-based application (www.randomization.com). In the intervention group $(n=25)$, the patients received an SNB with an infusion of $0.125 \%$ bupivacaine $(20 \mathrm{ml})$ and dexamethasone $(5 \mathrm{mg})$. As to the control group $(n=25)$, the patients received only a subcutaneous injection of $1 \%$ xylocaine $(1 \mathrm{ml})$ in the popliteal area without an SNB. The patients and assessors were blinded to the treatment allocations.

\section{Anesthetic protocol}

After being briefed on the research objectives and the experimental methods, each eligible patient signed a consent document on their respective day of admission. Two 
hours before surgery, each patient was given an oral premedication of paracetamol $(1000 \mathrm{mg})$. Following standard anesthetic monitoring (pulse oximetry, non-invasive blood pressure monitoring, and electrocardiogram), the patients were sedated using intravenous fentanyl (25-50 $\mathrm{mcg}$ ) and midazolam $(0.5-1 \mathrm{mg})$ before being administered oxygen through a nasal cannula $(3 \mathrm{~L} / \mathrm{min})$.

For both groups, an anesthesiologist specializing in regional anesthesia performed an ultrasound-guided ACB in the popliteal area, using $15 \mathrm{ml}$ of $0.33 \%$ bupivacaine. All patients were then placed in the lateral decubitus position. Both the control- and interventiongroup patients were administered a subcutaneous local infiltration of $1 \%$ xylocaine $(1 \mathrm{ml})$. However, the intervention-group patients subsequently also received an ultrasound-guided SNB (the popliteal approach); for that procedure, a mix of $0.125 \%$ bupivacaine $(20 \mathrm{ml})$ and dexamethasone $(5 \mathrm{mg})$ was used.

In the operating room, the patients received $2.0 \mathrm{ml}$ of spinal anesthesia with isobaric bupivacaine and an intravenous propofol infusion ( 25 to $50 \mathrm{mcg} / \mathrm{kg} / \mathrm{min}$ ) to achieve a mild to moderate sedation. At the end of the operation, all patients received PIA with $0.25 \%$ bupivacaine $(40 \mathrm{ml})$ and ketorolac $(30 \mathrm{mg})$. During the first three postoperative days, the standard oral postoperative regimen was followed for all patients: paracetamol (1000 mg every $6 \mathrm{~h}$ ), gabapentin ( $300 \mathrm{mg}$ before bedtime), and intravenous morphine for pain, as needed $(1 \mathrm{mg} /$ hour).

\section{Outcomes and measurement}

The primary outcome was the effects of using a combination of a low concentration of popliteal-SNB and dexamethasone as an adjunctive technique for TKA in patients susceptible to the adverse effects of NSAIDs on their pain scores during the first 24 postoperative hours. Postoperatively, the pain scores and motor function were determined by only one assessor, who was blinded to the group allocations. At 6, 12, 18, and $24 \mathrm{~h}$ after surgery, we assessed the postoperative pain scores at the anterior and posterior aspects of the knee while at rest and with motion (45 degrees of knee flexion). At the same times, the function of the tibialis anterior muscle was assessed and graded into 3 categories: 0 (normal); 1 (dorsiflexion, but less powerful than the untreated side); and $2=$ no strength to flex the ankle. Pain scores during physiotherapy were also recorded.

\section{Sample size}

Drawing upon data relating to adductor canal blocks and multimodal periarticular analgesia from work by Gwam et al. [7], a prospective power analysis revealed that 50 patients provided a $90 \%$ chance (power) to detect a reduction in visual-analog-scale score of 1.94 out of 10 during the first $24 \mathrm{~h}$ after surgery. The value of 1.94 represented a reduction from a moderate level of pain (scores 4-6) to a mild level (scores 1-3), e.g., from 4.94 in the control group to 3.00 in the SNB group. The type I error was 0.05 by an F-test; the standard deviation was not greater than 1.86 of the means; and a correction of the values for non-normal distribution was assumed.

\section{Statistical analysis}

All data were analyzed using IBM SPSS Statistics for Windows (version 24.0; IBM Corp., Armonk, NY, USA). The data were tested for normal distribution using histograms. Continuous data were presented as means and standard deviations (SD) or as medians and interquartile ranges (IQR), whereas categorical data were reported as numbers and percentages. A comparative analysis of the data of the 2 groups used the chi-squared test for group data, the unpaired t-test for continuous data with normal distribution, and the Mann-Whitney U test for continuous data with an abnormal distribution.

\section{Results}

In all, 63 patients who met the inclusion criteria were recruited. However, ten declined the invitation to join the study, while another three were not enrolled because of the unavailability of the researcher (Fig. 1 CONSORT 2010 flow diagram).

The 50 enrolled patients were divided into an intervention group and a control group (25 patients each). No significant differences were found in the age and sex distributions, body mass indexes, estimated glomerular filtration rates, underlying diseases, preoperative pain scores, and surgical and tourniquet times of the 2 groups (Table 1).

As to the postoperative pain scores of the groups (Table 2), there were significant differences in their pain scores at rest, pain scores on movement, and anteriorknee pain scores during the first $18 \mathrm{~h}$ after the surgery. The median (IQR) pain scores at rest at the 6-, 12-, and 18 -h timepoints were $1(0-4.5), 3(0-5)$, and $3(2-5)$ for the control group; and $0(0-0), 0(0-3)$, and $1(0-3)$ for the intervention group, respectively ( $p$-values $=0.012$, 0.021 , and 0.010 ). The median (IQR) pain scores on movement at 6,12 , and $18 \mathrm{~h}$ were $3(0-5.5), 5(2.5-6.5)$, and 7 (4-9) for the control group, and $0(0-1.5), 2(0-4)$ and $3(2-5)$ for the intervention group $(p$-values $=0.019$, 0.005 , and 0.001 ). The median (IQR) anterior-knee pain scores at 6,12 , and $18 \mathrm{~h}$ were $2(0-4.5), 2(0-4)$, and 3 $(0.5-5)$ for the control group, and $0(0-0), 0(0-3)$, and 1 $(0-3)$ for the intervention group ( $p$-values $=0.002,0.032$, and 0.015). 


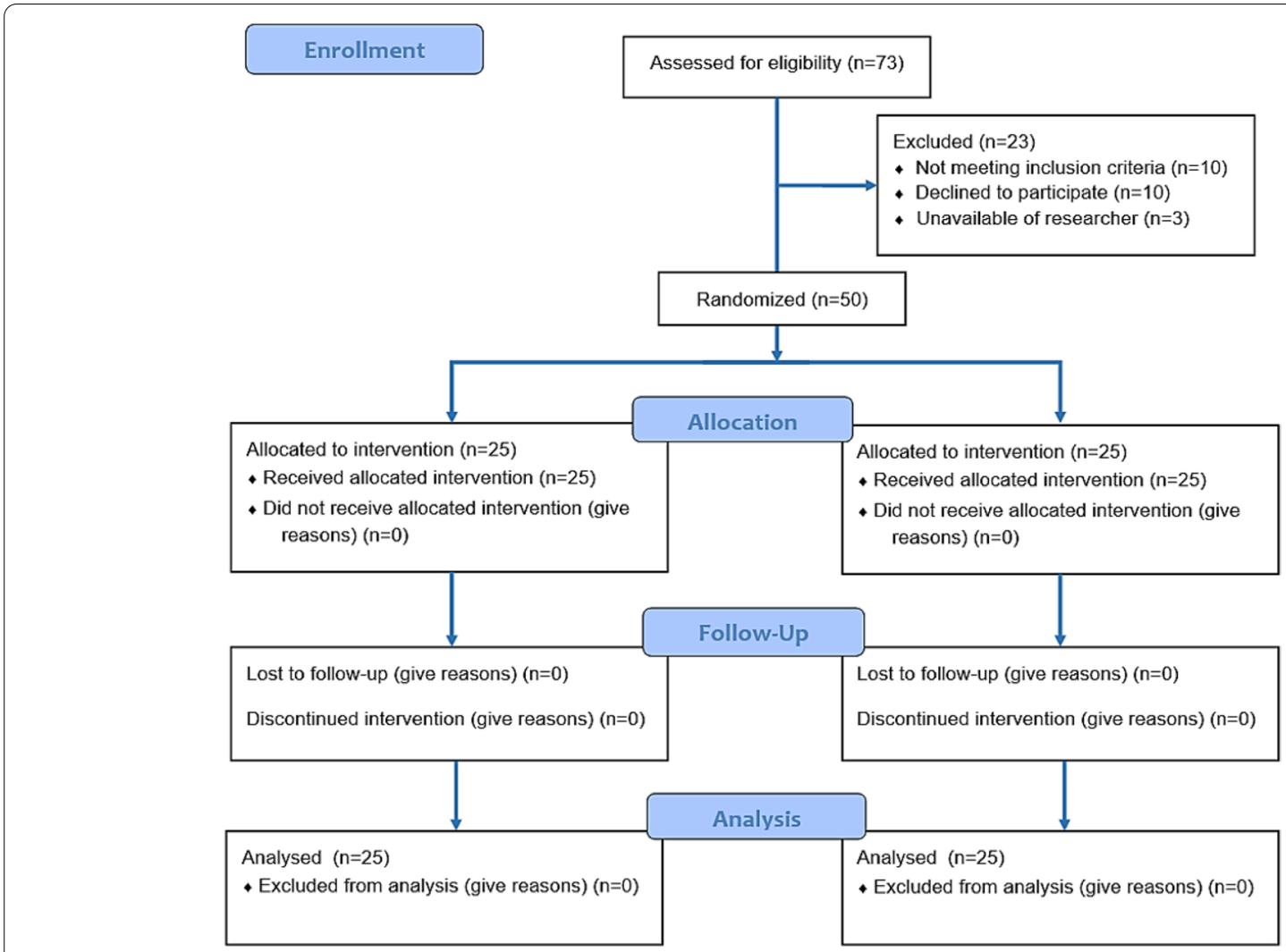

Fig. 1 CONSORT 2010 flow diagram

Table 1 Demographic data

\begin{tabular}{llll}
\hline & Control $(\boldsymbol{n}=\mathbf{2 5})$ & SNB $(\boldsymbol{n}=\mathbf{2 5})$ & P-value \\
\hline Female $(n, \%)$ & $18(72 \%)$ & $19(76 \%)$ & 0.747 \\
Age (mean \pm SD) & $73.88 \pm 7.76$ & $73.72 \pm 6.94$ & 0.939 \\
BMI (mean \pm SD) & $28.51 \pm 5.43$ & $26.28 \pm 3.00$ & \\
Underlying disease & & & \\
CKD (n, \%) & $17(68 \%)$ & $16(64 \%)$ & 0.080 \\
DM (n, \%) & $11(44 \%)$ & $13(52 \%)$ & 0.765 \\
HT (n, \%) & $21(84 \%)$ & $24(96 \%)$ & 0.571 \\
IHD (n, \%) & $7(28 \%)$ & $7(28 \%)$ & 0.349 \\
Stroke (n, \%) & $8(32 \%)$ & $6(24 \%)$ & 1.000 \\
eGFR (mean \pm SD) & $48.90 \pm 21.91$ & $52.94 \pm 22.13$ & 0.529 \\
Preoperative pain (median [IQR]) & $3(2-5)$ & $4(3-4.5)$ & 0.520 \\
Operative time (min) (mean \pm SD) & $90 \pm 21$ & $95 \pm 31$ & 0.835 \\
Tourniquet time (min) (mean \pm SD) & $85 \pm 17$ & $84 \pm 24$ & 0.462 \\
\hline
\end{tabular}

$B M I$ body mass index, $C K D$ chronic kidney disease, Control control group, $C r$ creatinine, DM diabetes mellitus, eGFR glomerular filtration rate, $H T$ hypertension, IHD ischemic heart disease, SNB popliteal-sciatic nerve block group

In contrast, a significant difference in the posterior pain scores was found only at the 12-h timepoint: the median (IQR) were $0(0-2.5)$ and $0(0-0)$ for the control and intervention groups, respectively $(p$-value $=0.038)$. There were also no significant differences in the motor function of the tibialis anterior muscle, the pain scores recorded during physiotherapy within $24 \mathrm{~h}$ and $48 \mathrm{~h}$, nor the levels of patient satisfaction of the 2 groups. 
Table 2 Postoperative results

\begin{tabular}{|c|c|c|c|c|c|}
\hline & \multicolumn{2}{|c|}{ Control $(n=25)$} & \multicolumn{2}{|c|}{ SNB $(n=25)$} & \multirow[t]{2}{*}{$P$-value } \\
\hline & Median & $(25-75)$ & Median & $(25-75)$ & \\
\hline \multicolumn{6}{|c|}{ Pain at rest $(N R S ; 0-10)$} \\
\hline $6 \mathrm{~h}$ & 1 & $0-4.5$ & 0 & $0-0$ & $0.012^{*}$ \\
\hline $12 \mathrm{~h}$ & 3 & $0-5$ & 0 & $0-3$ & $0.021^{*}$ \\
\hline $18 \mathrm{~h}$ & 3 & $2-5$ & 1 & $0-3$ & $0.010^{*}$ \\
\hline $24 \mathrm{~h}$ & 3 & $1-5$ & 2 & $1-3.5$ & 0.187 \\
\hline \multicolumn{6}{|c|}{ Pain on movement $(\mathrm{NRS} ; 0-10)$} \\
\hline $6 \mathrm{~h}$ & 3 & $0-5.5$ & 0 & $0-1.5$ & $0.019^{*}$ \\
\hline $12 \mathrm{~h}$ & 5 & $2.5-6.5$ & 2 & $0-4$ & $0.005^{*}$ \\
\hline $18 \mathrm{~h}$ & 7 & $4-9$ & 3 & $2-5$ & $0.001^{*}$ \\
\hline $24 \mathrm{~h}$ & 5 & $4.5-8$ & 5 & $2.5-7$ & 0.169 \\
\hline \multicolumn{6}{|c|}{ Anterior-knee pain (NRS; 0-10) } \\
\hline $6 \mathrm{~h}$ & 2 & $0-4.5$ & 0 & $0-0$ & $0.002^{*}$ \\
\hline $12 \mathrm{~h}$ & 2 & $0-4$ & 0 & $0-3$ & $0.032^{*}$ \\
\hline $18 \mathrm{~h}$ & 3 & $0.5-5$ & 1 & $0-3$ & $0.015^{*}$ \\
\hline $24 \mathrm{~h}$ & 3 & $1.5-5$ & 2 & $0-2.5$ & $0.028^{*}$ \\
\hline \multicolumn{6}{|c|}{ Posterior-knee pain (NRS; 0-10) } \\
\hline $6 \mathrm{~h}$ & 0 & $0-0$ & 0 & $0-0$ & 0.370 \\
\hline $12 \mathrm{~h}$ & 0 & $0-2.5$ & 0 & $0-0$ & $0.038^{*}$ \\
\hline $18 \mathrm{~h}$ & 0 & $0-2$ & 0 & $0-0$ & 0.129 \\
\hline $24 \mathrm{~h}$ & 0 & $0-2$ & 0 & $0-1$ & 0.666 \\
\hline \multicolumn{6}{|c|}{ Motor weakness ( $n, \%)$} \\
\hline $6 \mathrm{~h}$ & 4 & $16 \%$ & 6 & $24 \%$ & 0.615 \\
\hline $12 \mathrm{~h}$ & 2 & $8 \%$ & 2 & $8 \%$ & 1.000 \\
\hline $18 \mathrm{~h}$ & 1 & $4 \%$ & 1 & $4 \%$ & 1.000 \\
\hline $24 \mathrm{~h}$ & 1 & $4 \%$ & 0 & $0 \%$ & 1.000 \\
\hline \multicolumn{6}{|c|}{ Pain on physiotherapy (NRS; $0-10)$} \\
\hline $24 \mathrm{~h}$ & 6 & $5-8$ & 5 & $3-7$ & 0.083 \\
\hline $48 \mathrm{~h}$ & 3.5 & $2.3-6.8$ & 3.5 & $2-5$ & 0.478 \\
\hline $\begin{array}{l}\text { Patient } \\
\text { satisfaction } \\
\text { score }(0-10)\end{array}$ & 10 & $8-10$ & 10 & $10-10$ & 0.085 \\
\hline $\begin{array}{l}\text { Total } \\
\text { morphine in } \\
24 \mathrm{~h}(\mathrm{mg})\end{array}$ & 4 & $2-8$ & 2 & $0-2.5$ & $0.005^{*}$ \\
\hline
\end{tabular}

(25-75) interquartile range 25,75 , Control control group, NRS numeric rating scale, SNB popliteal-sciatic nerve block group, ${ }^{*} P$-value $<0.05$

The total morphine consumption of the 2 groups differed significantly during the first $24 \mathrm{~h}$ after the TKAs (Table 2). The median (IQR) total morphine consumptions of the control and intervention groups were $4(2-8) \mathrm{mg}$ and $2(0-2.5) \mathrm{mg}(p$-value $=0.005)$. Nevertheless, there was no statistical difference in their hospital lengths of stay, with mean \pm SD values of $132 \pm 25 \mathrm{~h}$ and $128 \pm 25 \mathrm{~h}$ for the control and intervention groups, respectively ( $p$-value $=0.591)$.

\section{Discussion}

Multimodal analgesia is currently a standard treatment for pain control. Nonsteroidal anti-inflammatory drugs play an essential role in reducing opioid consumption and acute postoperative pain management in orthopedic surgeries, including TKA. However, some patients are subject to the adverse effects of NSAIDs when prescribed systemically due to their potential side effects. Therefore, systemic NSAIDs are usually avoided in these patients, leading to inadequate pain control postoperatively. Our study aimed to focus on postoperative pain control following TKA in these challenging patients by adding SNB to ACB and PIA blocks. To our knowledge, this is the first study in TKA focusing on the NSAIDs susceptible patients.

In this study, pain scores (both at rest and on movement), as well as 24-h morphine consumption, were significantly lower in the SNB group compared to the control group. In the previous studies, SNB was an effective rescue block in patients with severe posterior knee pain $[9,19]$. However, adopting such a rescue block might be problematic at wards with limited medical personnel and equipment. To the best of our knowledge, only a few studies were published in the literature evaluating the addition of SNB to ACB and PIA in pain management after TKA. Kampitak et al. reported that SNB utilizing $0.25 \%$ levobupivacaine $(15 \mathrm{ml})$ with an ACB and PIA decreased morphine consumption. Nevertheless, the SNB group demonstrated a statistical difference in its degree of pain reduction relative to an obturatornerve-block group [20]. The other study from the same group of authors also compared SNB $(0.25 \%$ levobupivacaine $15 \mathrm{ml}$ ) to the interspace between the popliteal artery and the capsule of the posterior knee (IPACK block) after TKA [16]. They reported a higher incidence of a motor blockade in the SNB group than IPACK, but the SNB group had a lower incidence of posterior knee pain. In both studies, SNBs were performed with a higher concentration of local anesthetic and included postoperative NSAIDs in the protocol. In comparison, our study used a low-dose SNB (20 ml of $0.125 \%$ bupivacaine) and dexamethasone $(5 \mathrm{mg})$ combined with PIA and ACB and revealed the effectiveness for post-TKA pain control without motor weakness. Despite no systemic NSAIDs given, low-dose SNB was still significantly decreased pain scores at rest, on motion, and anterior knee up to $18-24 \mathrm{~h}$ following TKA.

Although we primarily hypothesized that pain would be alleviated in the SNB group due to the analgesia in the sciatic nerve distribution, mainly to reduce posterior knee pain. However, the median posterior knee pain score was reduced only at $12 \mathrm{~h}$. One of the reasons would be our intention to use a low concentration of 
bupivacaine $(0.125 \%)$ for SNBs to limit local anesthetic doses. With this concentration, the patients might not be able to characterize the specific area of pain, particularly if the pain was mild. Secondly, only $5(20 \%)$ patients (1 in the SNB and 4 in the control group) reported moderate-severe posterior knee pain. Due to the small sample size, it might be challenging to demonstrate the statistical difference in posterior knee pain. The incidence of moderate-severe posterior knee pain in our study also correlates to the previous studies by Gi et al. and Kampitak et al. The authors reported that $10-30 \%$ of the patients underwent TKA with a femoral nerve block and PIA had posterior knee pain $[16,21]$. Therefore, the benefit from adding SNB to adductor canal block and PIA may not be solely explained by posterior knee pain reduction.

We also added dexamethasone $5 \mathrm{mg}$ to the $\mathrm{SNB}$, while the control group patients did not receive dexamethasone. It has been well-demonstrated that dexamethasone is an effective adjunct to other multimodal analgesia techniques for postoperative pain control in TKA, regardless of the routes of administration $[18,22-$ 24]. Although a meta-analysis by De Oliveira et al. had reported that low dose dexamethasone $(<0.1 \mathrm{mg} / \mathrm{kg})$ did not provide opioid-sparing effect or analgesia during the early postoperative period $(<4 \mathrm{~h})$, however, dexamethasone significantly reduced late pain scores $(24 \mathrm{~h})$ both at rest and on movement, even at low dose [24]. Since we compared pain scores at $6-24 \mathrm{~h}$ postoperatively, dexamethasone given in the SNB injections to prolong the duration of the block may potentially be a confounder in our study and caused better pain relief in the SNB group compared to the control.

Although the mean 24-h morphine consumption was statistically lower in the SNB group $(1.96 \pm 2 \mathrm{mg}$ Vs. $3.80 \pm 2.48 \mathrm{mg}$ ), the slight difference may have modest clinical effects. The overall morphine consumption in our study was relatively lower than that reported from the earlier studies in patients with TKA. The reason might be that the patient-controlled analgesia was not cooperated in our protocol for morphine administration. Additionally, in older studies, 24-h morphine consumption and pain score were relatively high because multimodal analgesia combined with ultrasound-guided peripheral nerve block technique had not yet been a standard of care [19, 25]. Among newer studies in which multimodal analgesia was utilized with improvement in peripheral nerve block technique and PIA, average total doses of 24-h morphine consumption also ranged only between 2 and $10 \mathrm{mg}$ [ 9 , $16,26]$. In our study, overall 24-h morphine consumption was considered low in both groups, and the difference was only approximately $2 \mathrm{mg}$ which may not have significant clinical effects. The combination of ACB and PIA had already provided substantial pain control for most patients even without the use of NSAIDs. Therefore, the further opioid-sparing benefit of SNB was not well determined.

The risks of bupivacaine toxicity when combining several blocks must be taken into account. We limited the total dose of bupivacaine for the peripheral nerve blocks not to exceed $1.7 \mathrm{mg} / \mathrm{kg}$ ( $75 \mathrm{mg}$ for $45 \mathrm{~kg}$ patient). Also, $100 \mathrm{mg}$ of PIA was a low range commonly used in other studies, $100-400 \mathrm{mg}$, due to minimal absorption at this injection site and was considered safe by several studies $[16,27,28]$. Additionally, the duration between performing the peripheral nerve blocks and the PIA was about $90-120 \mathrm{~min}$, resulting in a mismatch of the peak plasma concentration of bupivacaine. The peak plasma concentration of peripheral nerve block is at $60 \mathrm{~min}$ [29-31], while the peak plasma concentration of PIA is at $24-48 \mathrm{~h}$ [32]. Previous studies showed that the total plasma concentrations of the maximum recommended doses of bupivacaine used in the peripheral nerve block and the PIA remained below half of the described toxicity thresholds. The margin of safety was probably much broader than current standards or the manufacturer's recommended dose [29-32]. However, all patients were closely monitored for the local anesthetic systemic toxicity (LAST) because of high doses of the local anesthetic in this study, especially in low body weight patients.

There are some limitations to this study. First, this was a single-center study using a small sample size, which had led to limitations for the analysis of the difference in posterior knee pain. Secondly, we did not use patientcontrolled analgesia for administering morphine postoperatively, therefore, it might underrepresent the 24-h morphine dose. Third, NSAIDs was still used in this study as a single dose of ketorolac in PIA. Only systemic NSAIDs was omitted. Finally, dexamethasone added into the SNB for an extended duration of analgesia may be a potential confounder for better pain control in the SNB group.

\section{Conclusions}

For patients susceptible to the adverse effects of NSAIDs, adding a low concentration of SNB combined with dexamethasone is an effective adjunctive technique to $\mathrm{ACB}$ and PIA for acute postoperative pain control in TKA. It offers benefits in early postoperative pain control (especially pain on movement) without a significant motor blockade.

\footnotetext{
Abbreviations

SNB: Sciatic nerve block; TKA: Total knee arthroplasty; NSAID: Non-steroidal anti-inflammatory drug; ACB: Adductor canal block; PIA: Periarticular infiltration analgesia; SD: Standard deviation; IQR: Interquartile range; BMI: Body mass index; CKD: Chronic kidney disease; Cr: Creatinine; DM: Diabetes mellitus; eGFR: Glomerular filtration rate; HT: Hypertension; IHD: Ischemic heart disease;
} 
NRS: Numeric rating scale; COX-2 inhibitor: Cyclooxygenase-2 inhibitor; IPACK: Infiltration of the local anesthetic between the popliteal artery and capsule of the knee; LAST: Local anesthetic systemic toxicity.

\section{Acknowledgements}

The authors are indebted to Miss Julaporn Pooliam for assistance with the methodology, Miss Chusana Rungjindamai for her invaluable support with administrative work, and to Mr. David Park for thoroughly proofreading the manuscript.

\section{Authors' contributions}

BS: Project leadership, study design, analysis and interpretation of data, manuscript revision. KS: study design, data collection. TK: data collection, statistical analysis, manuscript writing. SP, ST and PH: data collection. All authors have read and approved the manuscript.

\section{Funding}

Not applicable.

\section{Availability of data and materials}

The datasets used and/or analyzed during the current study are available from the corresponding author on reasonable request.

\section{Declarations}

\section{Ethics approval and consent to participate}

This study was approved by Siriraj Institutional Review Board (SIRB) COA no. Si123/2018. Project approval was obtained prior to the initiation of the study. After being briefed on the research objectives and the experimental methods, each eligible patient signed a consent document on their respective day of admission.

\section{Consent for publication}

Not applicable.

\section{Competing interests}

The authors have no relevant conflicts of interest to declare.

Received: 13 April 2021 Accepted: 27 October 2021

Published online: 13 November 2021

\section{References}

1. Lamplot JD, Wagner ER, Manning DW. Multimodal pain management in total knee arthroplasty. A prospective randomized controlled trial. J Arthroplast. 2014;29:329-34. https://doi.org/10.1016/j.arth.2013.06.005.

2. Roberts SL, Stout A, Dreyfuss P. Review of Knee Joint Innervation: Implications for Diagnostic Blocks and Radiofrequency Ablation. Pain Med (United States). 2020;21:922-38. https://doi.org/10.1093/PM/PNZ189.

3. Bendtsen TF, Moriggl B, Chan V, Børglum J. The optimal analgesic block for Total knee Arthroplasty. Reg Anesth Pain Med. 2016;41:711-9. https:// doi.org/10.1097/AAP.0000000000000485.

4. Nader A, Kendall MC, Manning DW, Beal M, Rahangdale R, Dekker R, et al. Single-dose Adductor Canal block with local infiltrative analgesia compared with local infiltrate analgesia after Total knee Arthroplasty: a randomized, double-blind, Placebo-Controlled Trial. Reg Anesth Pain Med. 2016;41:678-84. https://doi.org/10.1097/AAP.0000000000000494.

5. Sogbein OA, Sondekoppam RV, Bryant D, Johnston DF, Vasarhelyi EM, Macdonald S, et al. Ultrasound-guided motor-sparing knee blocks for postoperative analgesia following Total knee Arthroplasty. J Bone Jt Surg Am Vol. 2017:99:1274-81.

6. Koh HJ, Koh IJ, Kim MS, Choi KY, Jo HU, In Y. Does patient perception differ following Adductor Canal block and femoral nerve block in Total knee Arthroplasty? A simultaneous bilateral randomized study. J Arthroplast. 2017:32:1856-61. https://doi.org/10.1016/j.arth.2017.01.025.

7. Gwam CU, Mistry JB, Khlopas A, Chughtai M, Thomas M, Mont MA, et al. Does addition of multimodal Periarticular analgesia to Adductor Canal block improve lengths of stay, pain, discharge status, and opioid use after Total knee Arthroplasty? J Arthroplast. 2017;32:1470-3.
8. Andersen $\mathrm{L}$, Kehlet $\mathrm{H}$. Analgesic efficacy of local infiltration analgesia in hip and knee arthroplasty: a systematic review. Br J Anaesth. 2014:113:360-74.

9. Andersen KV, Nikolajsen L, Haraldsted V, Odgaard A, Søballe K. Local infiltration analgesia for total knee arthroplasty: should ketorolac be added? Br J Anaesth. 2013;111:242-8. https://doi.org/10.1093/bja/ aet030.

10. Rømsing J, Møiniche S, Østergaard D, Dahl JB. Local infiltration with NSAIDs for postoperative analgesia: evidence for a peripheral analgesic action. Acta Anaesthesiol Scand. 2000;44:672-83. https://doi.org/10. 1034/j.1399-6576.2000.440607.x.

11. Xiao X, Zhang Q, Ouyang Z, Guo X. Comparison of perioperative flurbiprofen axetil or celecoxib administration for pain management after total-knee arthroplasty: A retrospective study. Med (United States). 2018;97. https://doi.org/10.1097/MD.0000000000012391.

12. Lin J, Zhang L, Yang H. Perioperative Administration of Selective Cyclooxygenase-2 inhibitors for postoperative pain Management in Patients after Total Knee Arthroplasty. J Arthroplasty. 2013;28. https:// doi.org/10.1016/j.arth.2012.04.008.

13. Buvanendran A, Kroin JS, Tuman KJ, Lubenow TR, Elmofty D, Moric M, et al. Effects of perioperative Administration of a Selective Cyclooxygenase 2 inhibitor on pain management and recovery of function after knee replacement: a randomized controlled trial. J Am Med Assoc. 2003;290:2411-8. https://doi.org/10.1001/jama.290.18.2411.

14. Abdallah FW, Chan VWS, Gandhi R, Koshkin A, Abbas S, Brull R. The analgesic effects of proximal, distal, or no sciatic nerve block on posterior knee pain after total knee arthroplasty: a double-blind placebocontrolled randomized trial. Anesthesiology. 2014;121:1302-10.

15. Zorrilla-Vaca A, Li J. The role of sciatic nerve block to complement femoral nerve block in total knee arthroplasty: a meta-analysis of randomized controlled trials. J Anesth. 2018;32:341-50. https://doi.org/ 10.1007/s00540-018-2480-1.

16. Kampitak W, Tanavalee A, Ngarmukos S, Tantavisut S. Motor-sparing effect of iPACK (interspace between the popliteal artery and capsule of the posterior knee) block versus tibial nerve block after total knee arthroplasty: a randomized controlled trial. Reg Anesth Pain Med. 2020;45:267-76. https://doi.org/10.1136/rapm-2019-100895.

17. Albrecht E, Kern C, Kirkham KR. A systematic review and meta-analysis of perineural dexamethasone for peripheral nerve blocks. Anaesthesia. 2015;70:71-83. https://doi.org/10.1111/anae.12823.

18. Chong MA, Berbenetz NM, Lin C, Singh S. Perineural versus intravenous dexamethasone as an adjuvant for peripheral nerve blocks: a systematic review and Meta-analysis. Reg Anesth Pain Med. 2017;42:319-26. https://doi.org/10.1097/AAP.0000000000000571.

19. Perlas A, Kirkham KR, Billing R, Tse C, Brull R, Gandhi R, et al. The impact of analgesic modality on early ambulation following total knee arthroplasty. Reg Anesth Pain Med. 2013;38:334-9. https://doi.org/10.1097/ AAP.0b013e318296b6a0.

20. Kampitak W, Tanavalee A, Ngarmukos S, Amarase C. Opioid-sparing analgesia and enhanced recovery after Total knee Arthroplasty using combined triple nerve blocks with local infiltration analgesia. J Arthroplast. 2019;34:295-302. https://doi.org/10.1016/j.arth.2018.10.009.

21. Gi E, Yamauchi M, Yamakage M, Kikuchi C, Shimizu H, Okada Y, et al. Effects of local infiltration analgesia for posterior knee pain after total knee arthroplasty: comparison with sciatic nerve block. J Anesth. 2014;28:696-701. https://doi.org/10.1007/s00540-014-1793-y.

22. Tammachote N, Kanitnate S. Intravenous dexamethasone injection reduces pain from 12 to 21 hours after Total knee Arthroplasty: a double-blind, randomized, Placebo-Controlled Trial. J Arthroplasty. 2020;35:394-400. https://doi.org/10.1016/j.arth.2019.09.002.

23. Waldron NH, Jones CA, Gan TJ, Allen TK, Habib AS. Impact of perioperative dexamethasone on postoperative analgesia and side-effects: systematic review and meta-analysis. Br J Anaesth. 2013;110:191-200. https://doi.org/10.1093/bja/aes431.

24. De Oliveira GS, Almeida MD, Benzon HT, McCarthy RJ. Perioperative single dose systemic dexamethasone for postoperative pain: a metaanalysis of randomized controlled trials. Anesthesiology. 2011;115:57588. https://doi.org/10.1097/ALN.0b013e31822a24c2.

25. Hunt KJ, Bourne MH, Mariani EM. Single-injection femoral and sciatic nerve blocks for pain control after Total knee Arthroplasty. J Arthroplast. 2009;24:533-8. 
26. Yuenyongviwat V, Pornrattanamaneewong C, Chinachoti T, Chareancholvanich K. Periarticular injection with bupivacaine for postoperative pain control in Total knee replacement: a prospective randomized doubleblind controlled trial. Adv Orthop. 2012;2012:1-6.

27. Kim DH, Beathe JC, Lin Y, YaDeau JT, Maalouf DB, Goytizolo E, et al. Addition of infiltration between the popliteal artery and the capsule of the posterior knee and Adductor Canal block to Periarticular injection enhances postoperative pain control in Total knee Arthroplasty: a randomized controlled trial. Anesth Analg. 2019:129:526-35.

28. Nagafuchi M, Sato T, Sakuma T, Uematsu A, Hayashi H, Tanikawa H, et al. Femoral nerve block-sciatic nerve block vs femoral nerve blocklocal infiltration analgesia for total knee arthroplasty: a randomized controlled trial. BMC Anesthesiol. 2015;15:182. https://doi.org/10.1186/ s12871-015-0160-3.

29. Lapmahapaisan S, Chinachoti T, Maisat W, Kleebchan N, Chatsiricharoenkul S, Charoencholvanich K, et al. Plasma concentrations of bupivacaine after spinal anesthesia with single shot femoral nerve block and periarticular injection in total knee arthroplasty. J Med Assoc Thai. 2014;97:724-9.
30. De Leeuw MA, Bet PM, Wilhelm AJ, Vos RM, Hoeksema M, De Lange JJ, et al. Total plasma concentrations of bupivacaine, levobupivacaine and ropivacaine after combined psoas compartment-sciatic nerve block. Eur J Anaesthesiol. 2009;26:1083-5. https://doi.org/10.1097/EJA.0b013e3283 $308 \mathrm{e} 07$.

31. Misra U, Pridie AK, Mcclymont C, Bower S. Plasma concentrations of bupivacaine following combined sciatic and femoral 3 in 1 nerve blocks in open knee surgery. Br J Anaesth. 1991;66:310-3. https://doi.org/10.1093/ $\mathrm{bja} / 66.3 .310$.

32. Buys MJ, Murphy MF, Warrick CM, Pace NL, Gililland JM, Pelt CE, et al. Serum bupivacaine concentration after Periarticular injection with a mixture of liposomal bupivacaine and bupivacaine $\mathrm{HCl}$ during Total knee Arthroplasty. Reg Anesth Pain Med. 2017:42:582-7. https://doi.org/10. 1097/AAP.0000000000000636.

\section{Publisher's Note}

Springer Nature remains neutral with regard to jurisdictional claims in published maps and institutional affiliations.
Ready to submit your research? Choose BMC and benefit from:

- fast, convenient online submission

- thorough peer review by experienced researchers in your field

- rapid publication on acceptance

- support for research data, including large and complex data types

- gold Open Access which fosters wider collaboration and increased citations

- maximum visibility for your research: over $100 \mathrm{M}$ website views per year

At BMC, research is always in progress.

Learn more biomedcentral.com/submissions 